\title{
Neuroethics, the essential partner of neuroscience to face the burden of brain disorders
}

\section{Leonardi}

Director Neurology, Public Health, Disability Unit and Coma Research Centre, Fondazione IRCCS

Istituto Neurologico Carlo Besta, Milan, Italy

E-mail: Matilde.leonardi@istituto-besta.it

According to the 2016 Global Burden of Disease (GBD) study, the top 25 causes of disability-adjusted life years, DALY, a summary measure of health accounting for mortality and healthy years of life lost to disability, include brain disorders such as depressive disorders, self-harm, Alzheimer's disease, stroke and migraine (GBD2015 Collaborators, 2016). The brain is the most complex organ of the human being and represents one of the last truly great frontiers of science as it provides and controls virtually every function of the body including rational thinking, emotions, heartbeat, breathing, food and fluid intake, sex and the spectrum of disorders of the brain is large, covering hundreds of disorders that are listed in either the mental or neurological disorder chapters of the International Classification of Diseases ICD. Disorders of the brain have a high prevalence as well as short- and long-term impairments and disabilities are more complicated to analyze, diagnose and treat than other diseases (Morris et al. 2016). The early onset of most neuropsychiatric disorders, and their chronic or recurrent courses, makes this group of disorders the greatest contributor to disability, social and economic loss worldwide (Whiteford et al. 2013).

With an increase aging population, the prevalence of the most common neurological and psychiatric disorders will in fact dramatically increase and we have not found cures or means to delay or reduce their burden and economic impact, as well as the burden of care-givers and the loss of productivity and employment associated with this. Health economists have calculated that brain disorders may cost as much as $45 \%$ of the annual health budget of Europe, totaling around Euro 800 billion (Di Luca, Olesen 2014; Morris et al. 2016). From a position of relatively limited knowledge about the brain some 50 years ago, our basic understanding of the nervous system has expanded markedly since then. Indeed, basic and clinical neuroscientists have played a major part in this success story. The availability of astonishing new technologies, particularly over the last ten years, has greatly supported this development. Ethical questions related to the treatment of those who suffer from neurological and mental illness have been an issue since the beginning of medicine. Similarly, also the scientific investigation into the psychological and biological determinants of human behavior in morally relevant contexts has a long history. However, it was only with the rise of neuroscience as one of the leading scientific disciplines of the twenty-first century and the simultaneous development of new technology to investigate and control brain mechanisms that scientists, philosophers and bioethicists saw the need for a field of research and reflection they called neuroethics. 
Neuroethics is not just another branch of applied ethics. There are several reasons why this is true. For one thing, its scope is broader, encompassing not only concerns about the permissibility or advisability of using certain technologies to "read" minds, enhance capacities, or control behavior (all questions that are closely analogous to those pursued in bioethics) but also questions about what it means to be human, whether we have free will, the nature of knowledge and self-knowledge: questions that are more traditionally the terrain of philosophy rather than of applied ethics. For the other questions about acceptable degrees of risk, necessary levels of antecedent evidence before conducting human trials, consent capacity, and the tension between data sharing and privacy also have crucial importance in science. These issues can be more appropriate in the context of neuroscience research with novel neurotechnologies, as compared with other areas of biomedicine. For instance, concerns are heightened when considering acceptable degrees of risk for people receiving experimental deep brain stimulation to treat intractable mental illness, given that such interventions in the brain may affect the patient's mind. In other instances, ethical issues beyond the scope of traditional bioethics may arise because the brain is the organ of the mind and, for many, is believed to be the seat of the self. For example, emphasizing a mechanistic explanation of brain function may conflict with societal conceptions of personhood, individuality, and free will. How do we think about interventions, drugs, or devices that change cognition or change personality or issues of cognitive enhancement, drugs and interventions that affect a person's identity; brain privacy that is revealed by new neuroimaging techniques are among the challenging areas that neuroethics is exploring. Usually, the beginning of neuroethics as a discipline is regarded as linked to a series of interdisciplinary conferences bringing together neuroscientists, philosophers, policymakers and bioethicists that took place in several places in the UK and the USA (Roskies, 2002). In particular, the conference "Neuroethics: Mapping the Field" held on May 2002 in San Francisco received widespread attention and is considered one of the key milestones for the start of modern neuroethics research and resulted in a book, Neuroethics: Mapping the Field, edited by Steven J. Marcus and published by Dana Press (Farah Martha 2002).
However even though the rapid development of knowledge on the brain and the biology of the human mind made a systematic approach toward its ethical, legal, and social consequences exceptionally pressing since the end of last century, there is in any case evidence that the label "neuroethics" appeared in the contexts of neuroscience, medical ethics and philosophy of mind long before 2002 . (Roskies, 2002). This suggests that what is neuroethics today has historical progenitors in debates from very different disciplinary fields.

Neuroethics, as the International Society of Neuroethics defines is "the field that studies the implications of neuroscience for human self-understanding, ethics, and policy". (https://www.neuroethicssociety.org/what-isneuroethics). Neuroethics then is an essential partner to neuroscience, serving to anticipate and address ethical questions raised by neuroscience research, and to help guide the progress of the field. In our modern interconnected world, international collaboration in neuroscience and neuroethics is essential to realize the transformative potential of understanding brain function, and to address the implications for human selfunderstanding and society through a cross-cultural lens. Neuroethics has emerged as an interdisciplinary endeavor methodologically connecting diverse scientific and philosophical approaches only in subsequence of the growing knowledge in neuroscience and the associated promise of linking the subjective and personal world of experience and thought to the objective world of scientific data. Because of this, neuroethics has to be considered as a developing research field and not as an established discipline with clearly defined goals and methods. Despite the growing organization of neuroethics by scientific journals and research organization, the need for neuroethics research has not yet translated into a corresponding global research infrastructure. Neuroethics was formed primarily inside the established structures of the biomedical sciences and bioethics and only to a much smaller extent in the traditional humanities and the social sciences. This characterization as an emerging field of research has made the demarcation of neuroethics from adjoining disciplines an important issue ever since the field's official birth (Roskies, 2002; Cabrera, 2011; Levy, 2011). 
Roskies distinguishes two branches of neuroethics, which she refers to as the ethics of neuroscience and the neuroscience of ethics. The ethics of neuroscience is neuroethics as applied ethics; it consists of ethical reflection on neuroscience, its practice, and the technologies to which it gives rise. The neuroscience of ethics is instead concerned with what the sciences of the mind can tell us about the nature of morality and morally relevant topics in philosophy. In its guise as the neuroscience of ethics, one of the topics of neuroethics is how intuitions are generated. What brain regions are involved and how do they function to bring the agent to think that a particular action is forbidden, permissible, or obligatory? One of the most interesting findings produced by this work is that not all intuitions are generated in precisely the same manner. Instead, different processes are involved in generating different intuitions. (Roskies, 2002). Neuroethics thus is not just another branch of applied ethics. There are several reasons why this is true. For one thing, its scope is broader, encompassing not only concerns about the permissibility or advisability of using certain technologies to "read" minds, enhance capacities, or control behavior (all questions that are closely analogous to those pursued in bioethics) but also questions about what it means to be human, whether we have free will, the nature of knowledge and of self-knowledge: questions that are more traditionally the terrain of philosophy broadly conceived, rather than of applied ethics.

In the bibliometric analysis performed by Leefmann and colleagues (Leefmann et al. 2016) they found three tightly interconnected groups of thematically strongly interrelated subject-categories that can be seen as mapping the three perspectives on neuroethics. The first is related to Brain stimulation, the treatment used for neurodegenerative disorders such as Parkinson's disease and other neurodegenerative and psychiatric diseases. The second group is related to Enhancement, Psychopharmacology, and Addiction, issues that are frequently discussed in relation with each other and where the neuroethics discussion on cognitive enhancement is driven by phenomena such as the of offlabel use of prescription drugs such as Ritalin and Prozac. Finally, the third group is quite broad and is including empirical and theoretical issues such as Moral Theory, Social and economic neuroscience, Philosophy of mind and consciousness and Neuroimaging. What the authors observe is that the more approaches to neuroethics are theoretical the more hardly they connect with categories relevant for health-care and technologydriven approaches to neuroethics. For example, in the areas of Brain Death and Severe Disorders of Consciousness they found that new theoretical approaches in moral theory and in philosophy of mind are well informed by neuroscience research and by case studies that reveal knowledge about a wide range of consciousness phenomena. However, this empirical knowledge does not yet interrelate with the topics of practical neuroethics. This suggests that knowledgedriven approaches to neuroethics are currently still not dominant in the field (Leefmann et al.2016).

Increasingly, national governments across the globe are prioritizing investments in neuroscience and this is also closely linked to the ageing of population and to the increasing burden of brain disorders worldwide. Engaging with the underlying values and ethical concerns that drive brain research across cultural and continental divides is critical to future research. Culture influences what kinds of science are supported and where science can be conducted through ethical frameworks and evaluations of risk. Out of the several international initiatives that closely link neuroscience to neuroethics research here we focus on the European Human Brain Project (HBP) and on the recent U.S. Brain Research through Advancing Innovative Neurotechnologies (BRAIN) Initiative. Both have the potential to support neuroethics and thus neuroscience research so as that jointly they help to increasingly fight the rising burden of brain disorders, although results will have to be evaluated in the years to come.

The Human Brain Project (HBP) is a multi-national European brain research initiative to advance neuroscience and medicine and to create brain-inspired information technology. The HBP is funded by the European Commission Directorate General for Communications Networks, Content, and Technology (DG CONNECT) in the framework of the EU's Horizon 2020 research funding program and is one of the first two Future and Emerging Technologies (FET) Flagship projects. These were conceived to allow the EU to support larger, longer-term research efforts, with a view to generating significant innovation which would benefit citizens in Europe and further afield. Ethics and Society is part of the Human Brain Project's research core. 
Through its social and philosophical research, public engagement and ethics support, it promotes Responsible Research and Innovation (RRI) practices within the HBP and helps to shape the direction of the HBP in ethically sound ways that serve the public interest. Ethics and Society's research in the humanities is crucial for identifying and addressing the conceptual, social, ethical, legal and cultural implications and challenges raised by HBP research. The focus is on foresight, neuroethics and philosophy, public engagement, and researcher awareness. Ethics and Society also translate neuroethics research into practice by providing ethics support and implementing compliance programmes for the HBP (Amunts K. et al. 2016).

The U.S. Brain Research through Advancing Innovative Neurotechnologies (BRAIN) Initiative is an ambitious neuroscience research program that according to its founders aims to revolutionize the understanding of the human brain (Koroshetz et al., 2018). The National Institutes of Health (NIH) component of the Initiative is guided by BRAIN 2025: A Scientific Vision, a strategic plan developed with broad input from the scientific community

(https://www.braininitiative.nih.gov/strategic-

planning/brain-2025-report). While earlier approaches in neuroscience have often focused on the functioning of specific cell types or brain regions in isolation, BRAIN 2025 aims to foster innovation through investigating the dynamic interrelations of these varied components within circuits. BRAIN 2025 focuses on neural circuitry not only because of its critical role in brain function, but also because circuit dysfunction is the basis of the symptoms and disability in many neurological, mental, and substance use disorders. In many such disorders (including substance use and chronic pain), there is no evident brain pathology or lesion that can explain the grave disability experienced by afflicted persons. Progress in treatment for these conditions is likely to require a fuller understanding of the function of brain circuits. Even in the case of conditions such as stroke where discrete pathologies are known, treatment is limited by our abilities to monitor and precisely modulate brain circuit functions. Novel neurotechnologies may enable not only measurement of brain activity underlying thoughts and moods but also precise modulation of that activity, which will raise ethical, legal, and societal questions, including questions regarding how such interventions might affect autonomy, and who bears responsibility for such effects. (Bianchi 2018; Rommelfanger 2018). The BRAIN 2025 plan emphasizes accelerating technology development for neuroscience and applying these new tools and technologies to understand how dynamic patterns of activity in neural circuits are transformed into perception, emotion, cognition, and behavior in health and disease (Bianchi 2018).

To conclude as is for neuroscience, coordination across brain projects around the world in the space of neuroethics has the potential to strengthen our collective efforts through sharing lessons learned and best practices. Multinational partnerships invite new ideas and new approaches that may deepen our cross-cultural understanding of neuroscience advances and their ethical, legal, and social implications. This, in turn, maximizes the potential for international scientific collaboration and for increasing the benefits of neuroscience advances. Neuroethics is an essential partner to neuroscience, serving to anticipate and address ethical questions raised by neuroscience research, and to help guide the progress of the field. In our modern inter-connected world, international collaboration in neuroscience and neuroethics is essential to realize the transformative potential of understanding brain function, and to address the implications for human self-understanding and society through a cross-cultural lens. Partnering together neuroscience and neuroethics can contribute to the fight against the increasing burden of brain disorders.

\section{Conflict of Interest}

I have no conflict of interests and the opinions are of my own.

\section{References}

1. GBD2015 Mortality and Causes of Death Collaborators (2016). Global, regional, and national life expectancy, all-cause mortality, and cause-specific mortality for 249 causes of death, 1980- 2015: a systematic analysis for the Global Burden of Disease Study 2015. Lancet 2016 Oct 8; 388 (10053), 14591544. doi: 10.1016/S0140-6736(16)31012-1

2. Whiteford, H.A., Degenhardt, L., Rehm, J., Baxter, A.J., Ferrari, A.J., Erskine, H.E., Charlson, F.J., Norman, R.E., Flaxman, A.D., Johns, N., et al. (2013). Global burden of disease attributable to mental and substance use disorders: findings from the Global Burden of Disease Study 2010. Lancet 382, 1575-1586.

3. Di Luca, M. \& Olesen, J. (2014) The cost of brain diseases: a burden or a challenge? Neuron, 82, 1205-1208

4. Morris RGM, Oertel W, Gaebel W, Goodwin G, Little A, Montellano PA, Westphal M, Nutt DJ, Di Luca M (2016) 
Consensus Statement on European Brain Research: the need to expand brain research* in Europe - European Journal of Neuroscience,EJN pp. 1-8, 2016 doi:10.1111/ejn.13236

5. Farah Martha J (2002). Emerging Ethical issues in neuroscience. Nature Neurosceince 2001. 5(11):1123-1129. Doii: $10.1038 / \mathrm{nn} 1102-1123$

6. Neil Levy (2011) Neuroethics: A New Way of Doing Ethics, AJOB Neuroscience, 2:2, 3-9, DOI: 10.1080/21507740.2011.557683

7. Roskies, A. (2002). Neuroethics for the new millennium. Neuron 2002. Jul 3; 35: 21-23.

8. Cabrera, L. (2011). Neuroethics: a new way to do ethics or a new understanding of ethics? UABN 2, 25-26. doi: $10.1080 / 21507740.2011 .559921$

9. Leefmann, Jon; Levallois, Clement; Hildt, Elisabeth (2016) "Neuroethics 1995-2012. A Bibliometric Analysis of the Guiding Themes of an Emerging Research Field". Frontiers in Human Neuroscience. 10: 336. doi:10.3389/fnhum.2016.00336. ISSN 1662-5161. PMC 4929847. PMID 27445772.
10. Amunts K, Ebell C, Muller J, Telefont M, Knoll A, Lippert T. (2016) The Human Brain Project: creating a European research infrastructure to decode the human brain. Neuron. 2016; 92: 574581

11. Bianchi, D.W., Cooper, J.A., Gordon, J.A., Heemskerk, J., Hodes, R., Koob, G.F., Koroshetz, W.J., Shurtleff, D., Sieving, P.A., Volkow, N.D., et al. (2018). Neuroethics for the National Institutes of Health BRAIN Initiative. J. Neurosci. 38, 1058310585 .

12. Rommelfanger, K.S., Jeong, S.J., Ema, A., Fukushi, T., Kasai, K., Ramos, K.M., Salles, A., and Singh, I.; Global Neuroethics Summit Delegates (2018). Neuroethics questions to guide ethical research in the International Brain Initiatives. Neuron 100, 19 36. 